\title{
Coalescence of few layer graphene grains grown by chemical vapor deposition and their stacking sequence
}

\author{
Shumaila Karamat ${ }^{a}$ \\ Department of Physics, Middle East Technical University, Ankara 06800, Turkey; and Department of Physics, \\ COMSATS Institute of Information Technology, Islamabad 5400, Pakistan
}

Selda Sonuşen

Faculty of Engineering and Natural Sciences, Sabancı University, lstanbul 34956, Turkey

Munir Dede

NanoMagnetics Instruments Ltd., Ankara 06800, Turkey

Yigit Uysallı, Ekin Özgönül, and Ahmet Oral

Department of Physics, Middle East Technical University, Ankara 06800, Turkey

(Received 11 May 2015; accepted 27 October 2015)

\begin{abstract}
Few layer graphene is attractive due to its extraordinary electronic and optical properties, which are strongly influenced by the orientation between the layers called as stacking sequence. It is challenging to synthesize high quality large size single or multi layer graphene crystals on the metal catalyst using chemical vapor deposition technique. The present work is about synthesis of few layer graphene grains on platinum foil using ambient pressure chemical together vapor deposition technique. The main focus is how the different grains coalesced and maintain the stacking sequence. Different characterization techniques are used to analyze the grains when they are in the process of merging to make a bigger grain. Scanning electron microscopy clearly shows different stacking sequences and merging of different nucleation sites of different grains.

Interestingly, different stacking sequences are observed during the process of coalescence of grains. Raman spectroscopy gives accurate information about the number of layers and their stacking sequence. We observed Bernal $\mathrm{AB}$ and twisted layer stacking in the grains when they were combining together to grow into a bigger size. The full width at half maximum (FWHM) value of 2D Raman peaks appeared in the range of 52-69 $\mathrm{cm}^{-1}$ which shows an increase from the value of single layer graphene $\left(30.18 \mathrm{~cm}^{-1}\right)$ and identifies Bernal stacking in grains. For twisted stacking FWHM values lie in the range of $19-32 \mathrm{~cm}^{-1}$.
\end{abstract}

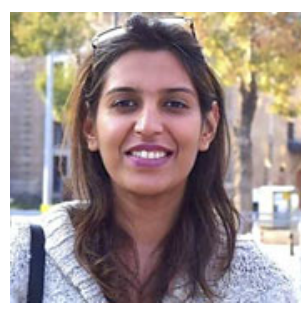

\begin{abstract}
Shumaila Karamat obtained her Ph.D. from Nanyang Technological University (Singapore) in 2011 where she worked in the area of dilute magnetic semiconductors. She then joined the group of Ahmet Oral under Marie-Curie cofunded postdoctoral fellowship at the Middle East Technical University (Ankara, Turkey). Since 2013, she is working as an experienced researcher and her work is focused on the fabrication of Scanning Hall Probe using graphene.
\end{abstract}

Shumaila Karamat

\section{INTRODUCTION}

Graphene, which consists of one atom thick sp2-bonded graphite, received the attention of different disciplines of physics because of its marvelous properties. ${ }^{1-4}$ To fully utilize the graphene in industry, fabrication of high quality

Contributing Editor: Mauricio Terrones

a) Address all correspondence to this author.

e-mail: shumailakaramat@gmail.com

DOI: $10.1557 / j m r .2015 .350$ graphene is essential. The exfoliated single-layer graphene exhibit carrier mobility of $\sim 10,000 \mathrm{~cm}^{2} / \mathrm{V} \mathrm{s}$ when transferred on $\mathrm{Si}$ wafer and $200,000 \mathrm{~cm}^{2} / \mathrm{V} \mathrm{s}$ in its suspended form but the area coverage and number of layers are not well controlled. The most promising method to grow large area graphene on metal catalysts is chemical vapor deposition (CVD).$^{5-7}$ The problem widely faced by the scientific community in the CVD growth is the presence of crystallographic graphene domains. The growth of graphene is initiated at different nucleation sites 
on the metal catalyst which give full coverage with the increase in growth time but also promote the growth of few layer grains having different crystallographic orientation. During the growth procedure, nucleation density of graphene plays an important role because it controls grain boundaries which would affect the quality of graphene. The material properties like mechanical strength, mobility, doping percentage, and thermal transport are greatly affected by these grain boundaries. ${ }^{8}$ The grown single layer graphene (SLG) grown via CVD mostly shows lower mobility $\sim 3000 \mathrm{~cm}^{2} / \mathrm{V}$ s as compare to mechanically exfoliated graphene. ${ }^{9}$ To consistently fabricate high quality graphene, there is still more to be understood about the growth parameters and the nucleation of domains.

Recently, attention has been paid to decrease the nucleation density of graphene by growing single layer crystalline grains of few millimetre in size. ${ }^{10}$ Large area grains were grown on metal catalysts like $\mathrm{Cu}^{10-13}$ and $\mathrm{Pt}^{14}$ using low and ambient pressure CVD (APCVD) technique, but there is no final recipe which can eliminate the nucleation sites. It is challenging to obtain a continuous layer of graphene without graphene domains using CVD. Effect of different parameters like catalyst annealing time ${ }^{11}$ precursor gas pressures, ${ }^{15,16}$ and growth temperature ${ }^{17}$ was widely studied for graphene domains grown on $\mathrm{Cu}$ using CVD but very few reports have been published for graphene grown on Pt. In materials, the process of nucleation, growth, and coalescence of grains breaks periodicity and exhibit polycrystalline structure. The grain boundaries impede dislocation motion in metals which mechanically strengthen them, but on the other side increases the electron scattering which affect the conductivity. Graphene researchers are concerned about the nucleation and the presence of graphene domains on catalyst surfaces which affect the growth and crystallinity of SLG. If nucleation sites would be located far away on the metal catalyst and the growth would take place quickly, then it is possible to grow large area SLG but growth must be isotropic to reduce defects in graphene. Recently, Gao et al. were successful in growing millimetre size graphene grains on Pt with high crystallinity. ${ }^{14}$ A lot of parameters still need to be studied for graphene growth on Pt. In this study, graphene via CVD was grown on $\mathrm{Pt}$ and the evolution of graphene grains from smaller to bigger size were observed. Further, a particular set of overlapped grains and their stacking sequences were investigated.

\section{EXPERIMENTS}

Graphene grains were grown on Pt foils in APCVD system using $\mathrm{CH}_{4}$ (99.995\% purity, Messer, Ankara, Turkey), $\mathrm{H}_{2}$ (99.999\%, BOS, Ankara, Turkey), and Argon (99.999\% purity, Messer) precursor gases. The graphene grains were grown at $1 \mathrm{sccm} \mathrm{CH}_{4}$ flow rate for $3 \mathrm{~h}$ at $1040{ }^{\circ} \mathrm{C}$. Initially, the Ar gas flowed at a rate of 300 sccm for $15 \mathrm{~min}$ to purge the system and the hydrogen gas was introduced afterward at the rate of $50 \mathrm{sccm}$. The hydrogen flow was maintained at $150 \mathrm{sccm}$ when the temperature reached $800{ }^{\circ} \mathrm{C}$ and was maintained at the end of the experiment. At $1040{ }^{\circ} \mathrm{C}, \mathrm{CH}_{4}$ was introduced into the system at a flow rate of $1 \mathrm{sccm}$ for $3 \mathrm{~h}$ to grow the graphene. After growth, the furnace was opened to cool down the samples quickly.

The graphene on Pt was coated with C9-PMMA electron beam resist to provide mechanical support during transfer. The graphene was transferred using a hydrogen bubbling technique, ${ }^{14}$ the hydrogen bubbles produced during electrolysis of lithium hydroxide reach toward PMMA/ graphene/Pt electrode and try to stick with Pt and as a result peel off graphene. The graphene/PMMA stack was scooped out and transferred to deionized (DI) water. After cleaning in water, the stack was transferred to $\mathrm{SiO}_{2}$ covered $\mathrm{Si}$ and left overnight in the air for drying. After drying, samples were heated at $180{ }^{\circ} \mathrm{C}$ on a hot plate followed by vacuum drying at $70{ }^{\circ} \mathrm{C}$ for $3 \mathrm{~h} .{ }^{15}$ The PMMA layer was dissolved in acetone and the sample was cleaned with methanol, followed by a nitrogen blow dry. Optical microscopes (Carl Zeiss Axio Scope A1 MaT, Jena, Germany, and Nikon Eclipse LV 100, Tokyo, Japan) were used to see the graphene domains and their evolution. The samples were also imaged using Zeiss Supra 35VP field emission scanning electron microscope (Carl Zeiss, Jena, Germany). Renishaw inVia Reflex microRaman spectrometer with $532 \mathrm{~nm}$ laser source is used to measure the Raman spectra.

\section{RESULTS AND DISCUSSION}

During CVD growth, hydrocarbon species react with the presence of metal thin foils due to high temperatures (900-1100 ${ }^{\circ} \mathrm{C}$ ). The metal catalyst plays an active role in the decomposition of hydrocarbon and the nucleation of carbon species. Different factors like carbon solubility in the metal catalyst, crystal structure of the catalyst, and the growth conditions play their role in the growth mechanism of graphene. The growth of graphene is due to the solubility of carbon species in the metal, but the solubility is different for different metal catalysts. For $\mathrm{Cu}$, the solubility of carbon is lower $(\sim 0.001$ at.\%) and the growth of graphene only takes place at the surface of the metal. For Pt, the solubility of carbon is higher $(\sim 0.9 \%)$ and the chances of graphene growth would be a combination of two processes i.e., diffusion and precipitation. ${ }^{18}$ The growth of graphene takes place due to carbon nucleation on Pt surface and the expansion of graphene grains in two dimensions. Graphene has very little interaction with the $\mathrm{Pt}$ and can easily expand and cross the grain boundaries of the metal. The stronger affinity of the carbon-carbon atoms helps them to be self assembled in $s p 2$ coordination and expand efficiently on the metal catalyst. Moreover, the weak interactions between the graphene and the $\mathrm{Pt}$ 
substrate causes the formation of rotational domains. ${ }^{19}$ Theoretically, the mismatch between Moire superstructure unit cell and Pt atoms causes the rotational domains and they are expected to occur frequently. ${ }^{20}$

Figure 1 shows optical images of different grains which are combining together. Figure 1(a) shows more nucleation sites on the Pt grain boundaries as compared to the plain area. The cause of abundant nucleation at the grain boundaries is the surface roughness of Pt metal which may increase due to repetitive use. Figures 1(b)-1(d) shows different grains combining together and expanding. We grow graphene for $3 \mathrm{~h}$ which allows the nucleation and growth of multiple grains and their coalesence with each other. It is well known that in CVD growth method, a second layer of graphene nucleate and grow under the first layer with the same nucleation site. ${ }^{21} \mathrm{~A}$ lot of understanding has been gained on the SLG formation where the first layer forms due to supersaturation of carbon gas adatoms. The adatom concentration is very important in growth, most of the carbon islands nucleate at defect sites on the metal catalyst.

The fully developed grains show Bernal and twisted stacking, however, the dominance of twisted stacking in fully developed grains were higher.

Figure 2 shows SEM images of single and coalesced graphene grains. The graphene grains were hexagonal in shape before coalescence as seen from the trend of other grains in the sample. The hexagonal grains exhibits corners of $120^{\circ}$, suggesting that their edges are parallel to specific crystallographic directions. It is worthy to note here that the hexagonal shape is different from the flower-like shape of graphite grains, which can be obtained by low pressure CVD. ${ }^{6,16}$ In APCVD hexagonal graphene grains form with straight edges. The edges of two graphene grains line up parallel during growth and one grain overlaps another. $^{22}$ The overlapping grain maintains the stacking of the two grains. ${ }^{23}$ In case of fewer grains, their edges line up parallel to each other and overlap another. It is desired that if somehow cystallographic orientations of neighboring domains align then it will give a continuous film which will surely decrease the detrimental effect of grain boundaries. In SEM images, the grain boundaries of the coalesced grains are not visible. To be sure about the removal of grain boundaries at the places where graphene grains are combining, we performed Raman mapping of different coalesced grains. Raman contour maps are plotted in Origin and Matlab and images are constructed from the intensities of $I_{\mathrm{G}}, I_{\mathrm{D}}$, and $I_{2 \mathrm{D}}$ Raman bands.

Raman spectroscopy has been used extensively to investigate the structural and electronic characteristics of graphitic materials. It provides useful information about the defects (D band), the stacking order (2D band) as well as in-plane vibration of $s p 2$ carbon atoms (G band). ${ }^{24-26}$ The Raman bands of graphene give a clear insight about different phenomena, like, the 2D band simply gives information about whether the graphene is single or multi layer by fitting multiple peaks due to splitting of electronic band structure. ${ }^{27,28}$ The general features of Raman spectrum for graphene lie in the range

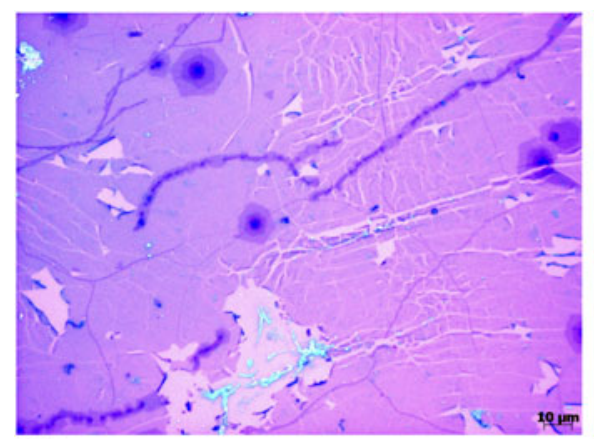

(a)

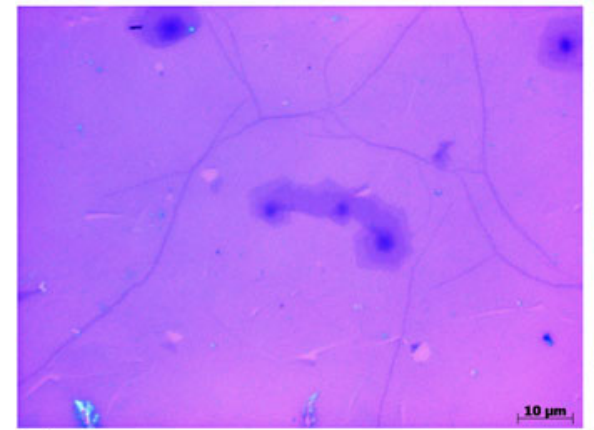

(c)

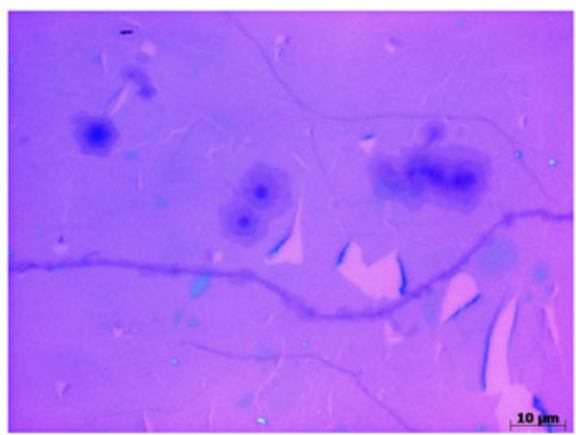

(b)

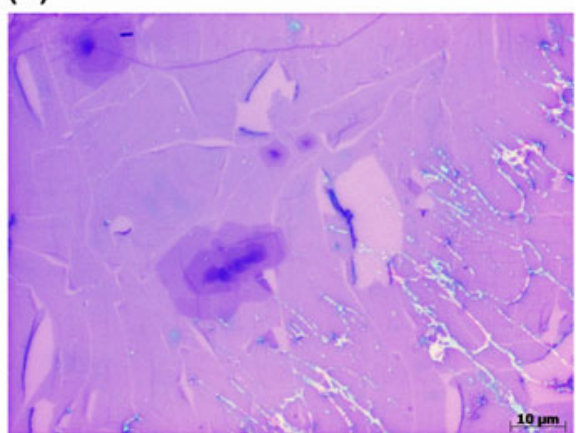

(d)

FIG. 1. (a) Optical image of individual graphene grains and chain of grains at metal boundaries, (b) optical image of two graphene grains coalesced together, (c) optical image of three graphene grains coalesced together and (d) optical image of the few grains coalesced together. 


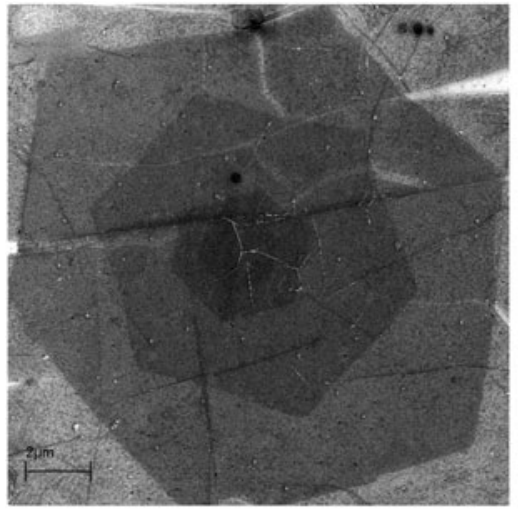

(a)

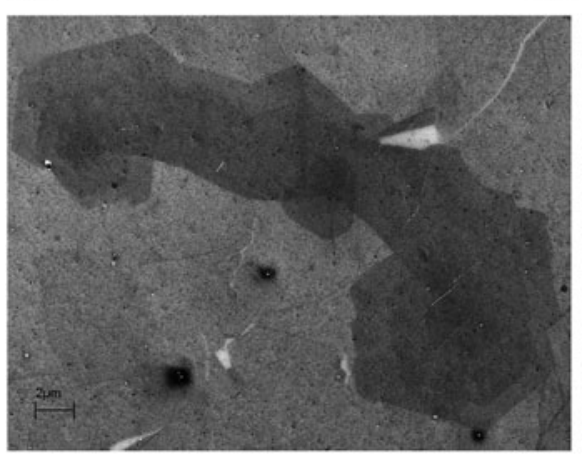

(c)

(b)
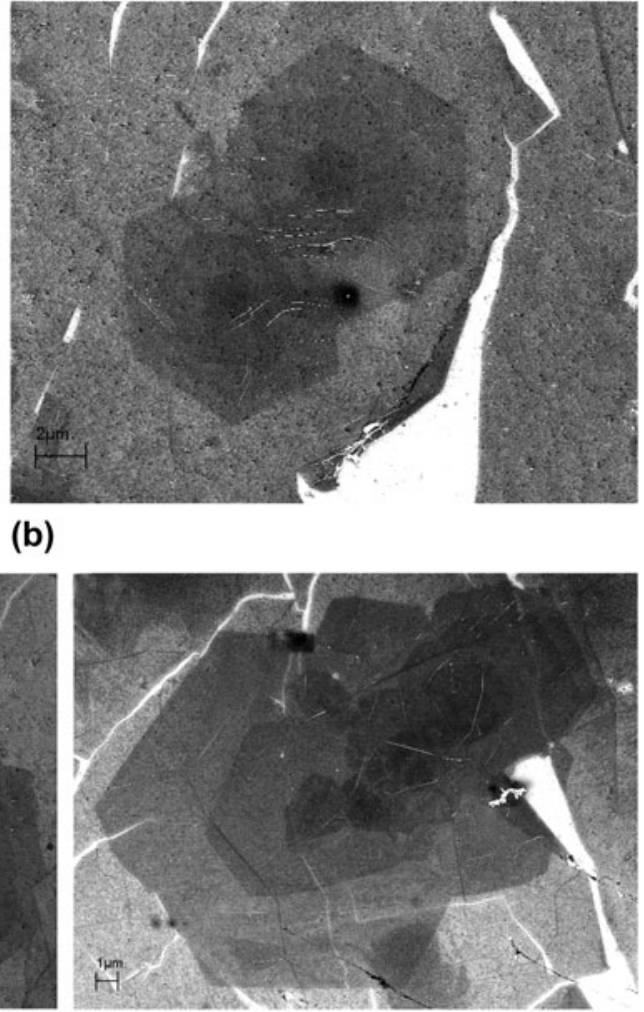

(d)

FIG. 2. (a) SEM image of graphene grain showed in Fig. 1(a), (b) SEM image of two graphene grains coalesced together, (c) SEM image of three graphene grains coalesced together and (d) SEM image of few grains coalesced together.

of $1000-3000 \mathrm{~cm}^{-1}$ with three characteristic bands D $\left(\sim 1350 \mathrm{~cm}^{-1}\right), \mathrm{G}\left(\sim 1580 \mathrm{~cm}^{-1}\right)$, and $2 \mathrm{D}\left(\sim 2670 \mathrm{~cm}^{-1}\right)$. The $\mathrm{D}$ band that originates from transverse optical phonon lies around the $K$ point of the Brillouin zone and activates in the presence of defects. ${ }^{29}$ The intensity of the $\mathrm{D}$ band gives information about the crystalline quality of a sample. ${ }^{30}$ The $\mathrm{G}$ band is a doubly degenerate phonon mode $\left(E_{2 \mathrm{~g}}\right.$ symmetry) at the Brillouin zone center and originates due to in-plane vibration of $s p 2$ bonded carbon atoms. The width of the $\mathrm{G}$ band gives information about the doping level of a sample. ${ }^{31}$ The $2 \mathrm{D}$ or $\mathrm{G}^{\prime}$ band is the second most prominent band in graphene after $\mathrm{D}$ band which arises from the scattering of two equal and opposite phonon wave vectors. In the first Brillouin zone, Raman double resonance phenomena involves two phonons and electronic states which lie near two nonequivalent $K$ points and create a 2D peak in the Raman spectrum. ${ }^{32,33}$ Moreover, a shift in the position of the $2 \mathrm{D}$ band gives information about the strain in the sample. $^{34}$

The electrons exhibit linear dispersion near Dirac $\mathrm{K}$ points and behave as massless Dirac fermions in graphene. ${ }^{35,36}$ In AB stacked bilayer graphene, the valence and conduction band splits into two parabolic nodes near the $\mathrm{K}$ point because of the interaction of $\mathrm{p}$ electrons and at this point electrons behave as massive Dirac fermions instead of massless Dirac fermions. ${ }^{36,37}$ Interestingly, the band gap of bilayer graphene system can be tuned by applying electric field biasing and the addition of few layers reveals variation in the electronic structure of graphene. ${ }^{38}$ Apart from AB stacking, twisted or arbitrary stacking also exhibits unique properties because of different crystalline structure and its interaction with $p$ electrons.

Figures 3-5 shows Raman spectrum of coalesced grains with their intensity maps. We observed a very small D peak in the samples which show good crystalline quality of the graphene layer. The most promising changes can be seen in the peak positions and full width at half maximum (FWHM) of 2D peak in different regions of coalesced grains which clearly show different number of layers. The change in position and width of $2 \mathrm{D}$ peak hints toward different stacking sequences in the coalesced grains which supports the findings of Rao et al. $^{23}$ that two grains, aligned parallel to each other, maintain the stacking sequence. In Fig. 3(a), optical micrograph was marked for different regions which shows single and few layer regions of grains merging together. According to double resonance model, four transitions are involved in the emergence of the $2 \mathrm{D}$ peak, 

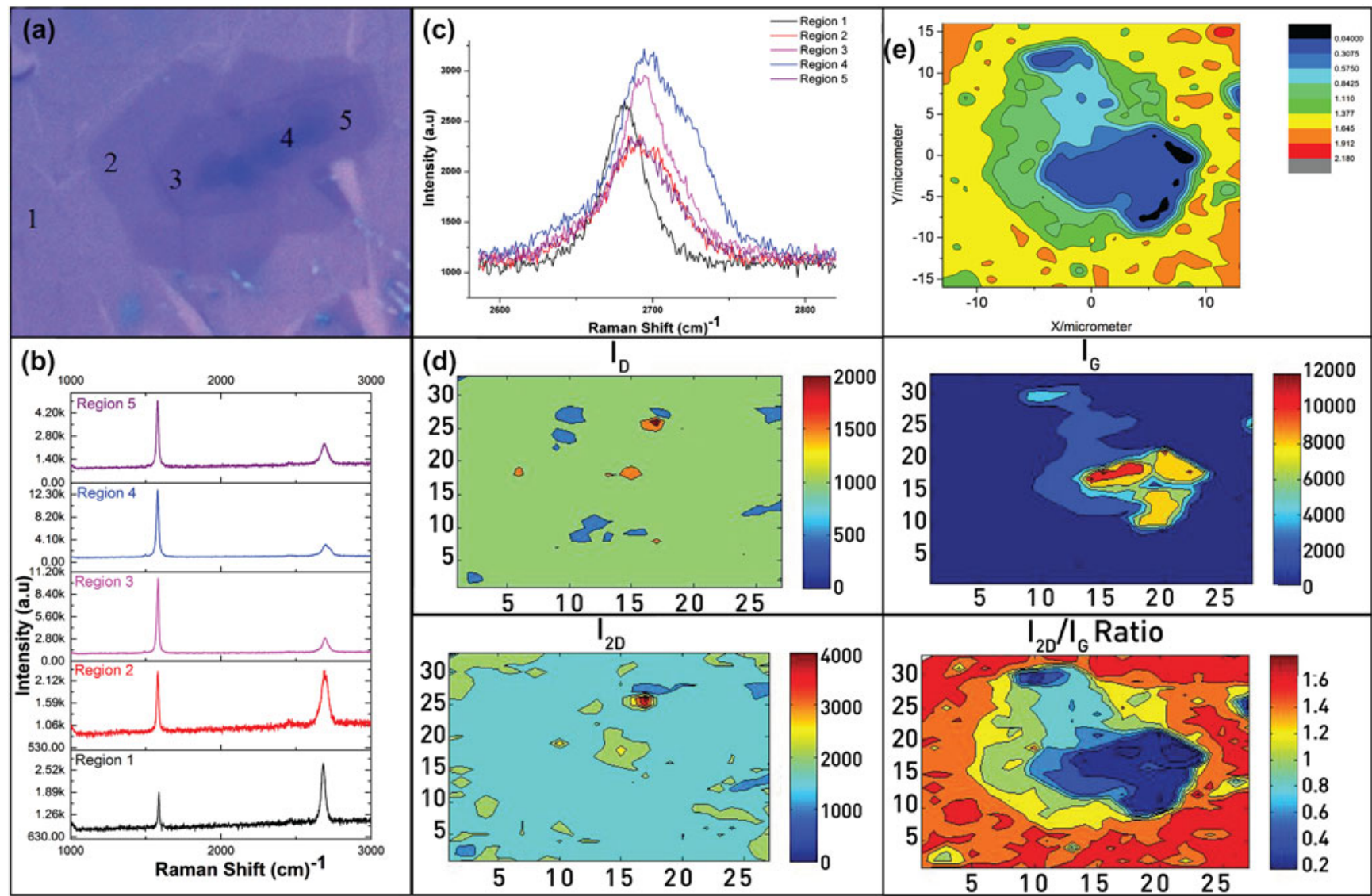

FIG. 3. (a) Marked optical image of graphene grain for Raman spectrum at different points, (b) Raman spectra for Bernal (AB) stack coalesced grains at regions $1,2,3,4$, and 5 , (c) $2 \mathrm{D}$ peak for all the regions, (d) intensity maps for $\mathrm{D}, \mathrm{G}$ and $2 \mathrm{D}$ peaks plotted in matlab and (e) $I_{2 \mathrm{D}} / I_{\mathrm{G}}$ ratio contour mapping in Origin 8.5 software.

i.e., excitation of electron-hole pair via a laser, scattering, backscattering of excited electron by two phonons, and electron hole-pair recombination. ${ }^{27}$ This process creates two phonons near $\mathrm{K}$ and $\mathrm{K}^{\prime}$ in the Brillouin zone and reflects evolution in the electronic band structure of graphene. For SLG, the 2D peak is symmetric and is fitted with one Lorentz peak which indicates that one Raman cycle is excited near the $K$ and $K^{\prime}$ points. ${ }^{39}$ The Raman spectrum of marked region 1 shows SLG with FWHM of $30.18 \mathrm{~cm}^{-1}$ located at $2681.84 \mathrm{~cm}^{-1}$ with an intensity ratio $\left(I_{2 \mathrm{D}} / I_{\mathrm{G}}\right)$ of 1.45 . The typical intensity of the $2 \mathrm{D}$ peak $\left(I_{2 \mathrm{D}}\right)$ is more than double of the intensity of G peak $\left(I_{\mathrm{G}}\right)$ for SLG and it has been observed at region 1. The Raman spectrum of region 2 shows broader FWHM of $55.43 \mathrm{~cm}^{-1}$ located around $2691.81 \mathrm{~cm}^{-1}$ with an intensity ratio $\left(I_{2 \mathrm{D}} / I_{\mathrm{G}}\right)$ of 1.01 . From the contrast of the optical micrograph, region 2 appears to be bilayer graphene but carefully examining the intensity ratio map shows that it is actually a trilayer graphene which is supported by the intensity ratio value, which is less than that of SLG. The FWHM for trilayer graphene appeared around $56.2 \pm 1.6 \mathrm{~cm}^{-1}$ (Ref. 39) and matches well with the region 2 FWHM of our samples.
For AB stacked bilayer system, the $\pi$ electrons split the valence and conduction bands into four parabolic bands $\left(\pi_{1}, \pi_{2}, \pi_{1}^{*}\right.$, and $\left.\pi_{2}{ }^{*}\right)$ near the K point, ${ }^{40}$ and two of them have higher relative intensities than the other two. ${ }^{27}$ The 2D peak shows dispersive behavior for bi-layer graphene and it would be fitted with four Lorentzian peaks with slightly different frequencies and the FWHM is almost twice than that of SLG. With the increase in number of graphene layers, there will be a significant decrease in the intensity of 2D peak components, the electronic bands split into complex configuration and the excited electronhole pair undergoes more scattering cycles as compared to bilayer graphene and it causes dispersion or widening in the Raman spectrum of the 2D peak for tri or tetra layers. ${ }^{39,40}$ The Raman spectrum for region 3 shows FWHM of $42.72 \mathrm{~cm}^{-1}$ observed around $2694.28 \mathrm{~cm}^{-1}$ with an intensity ratio $\left(I_{2 \mathrm{D}} / I_{\mathrm{G}}\right)$ of 0.28 . Optical micrograph, intensity ratio value and consistent increase in peak position hints toward tetra layer $\mathrm{AB}$ stacked graphene but the decrease in FWHM value hints toward the folding of graphene. ${ }^{39}$ The Raman spectrum for region 4 shows broader FWHM of $64.52 \mathrm{~cm}^{-1}$ observed around $2700.85 \mathrm{~cm}^{-1}$ with an intensity ratio $\left(I_{2 \mathrm{D}} / I_{\mathrm{G}}\right)$ of 0.23 and predict tetra layer graphene. ${ }^{39}$ The Raman 


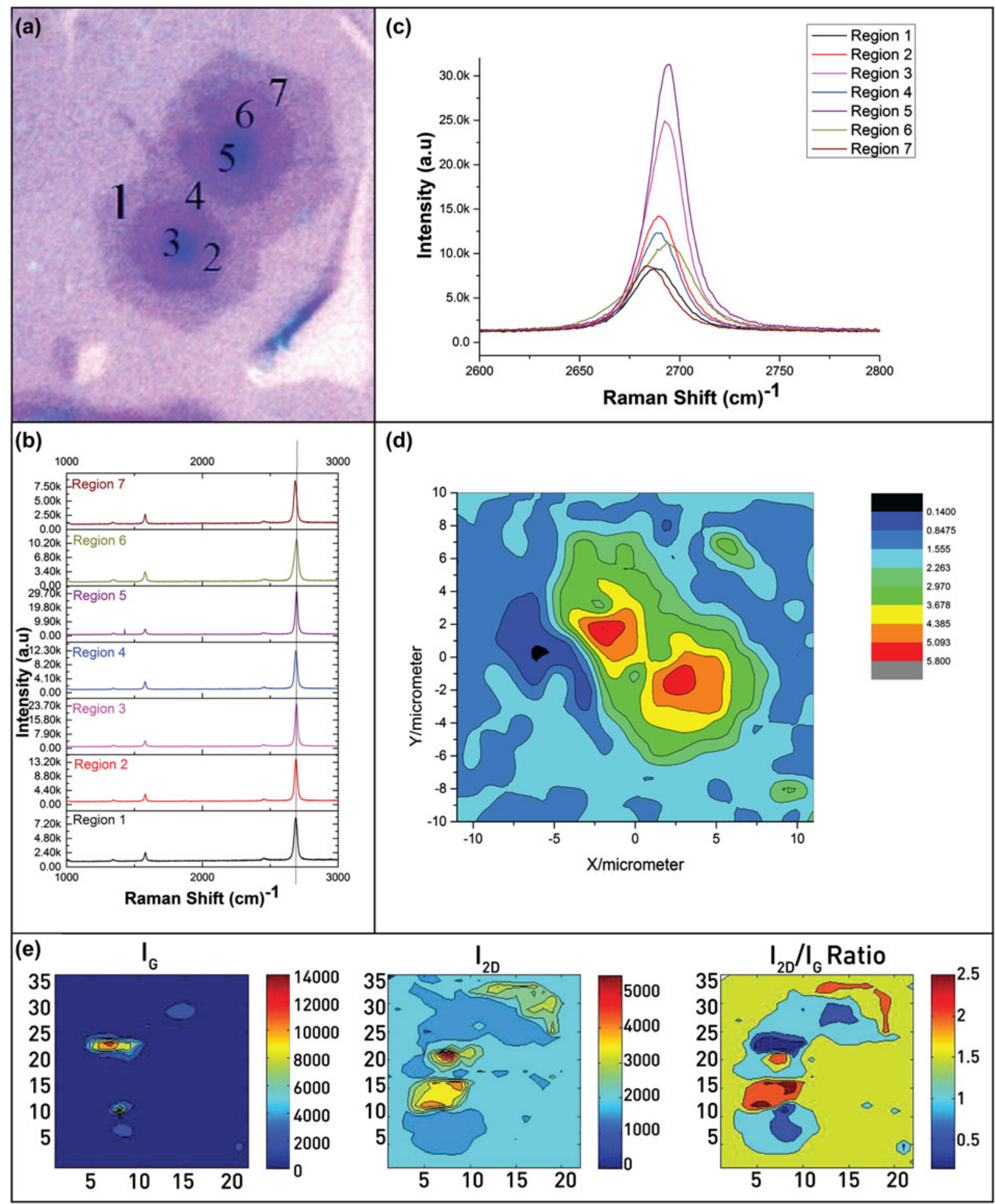

FIG. 4. (a) Marked optical image of graphene grains for Raman spectrum at different points, (b) Raman spectra for twisted stack coalesced grains at regions $1,2,3,4,5,6$, and 7 , (c) $2 \mathrm{D}$ peak for all the regions, (d) $I_{2 \mathrm{D}} / I_{\mathrm{G}}$ ratio contour mapping in Origin 8.5 software and (e) intensity maps for $\mathrm{G}$ and $2 \mathrm{D}$ peaks plotted in matlab.

spectrum for region 5 showed FWHM of $52.91 \mathrm{~cm}^{-1}$ observed around $2690.02 \mathrm{~cm}^{-1}$ with an intensity ratio $\left(I_{2 \mathrm{D}} / I_{\mathrm{G}}\right)$ of 0.46 hints bilayer graphene. ${ }^{39}$ Liu et al. ${ }^{41}$ observed FWHM values of the 2D peak in the range of
47.5-62.0 $\mathrm{cm}^{-1}$ for the $\mathrm{AB}$ stacked bilayer graphene having $I_{2 \mathrm{D}} / I_{\mathrm{G}}$ ratios in the range of $0.83-1.46$. Carefully analysing the contouring boundaries of the coalesced grains in Fig. 3(d), the values of FWHM for different 


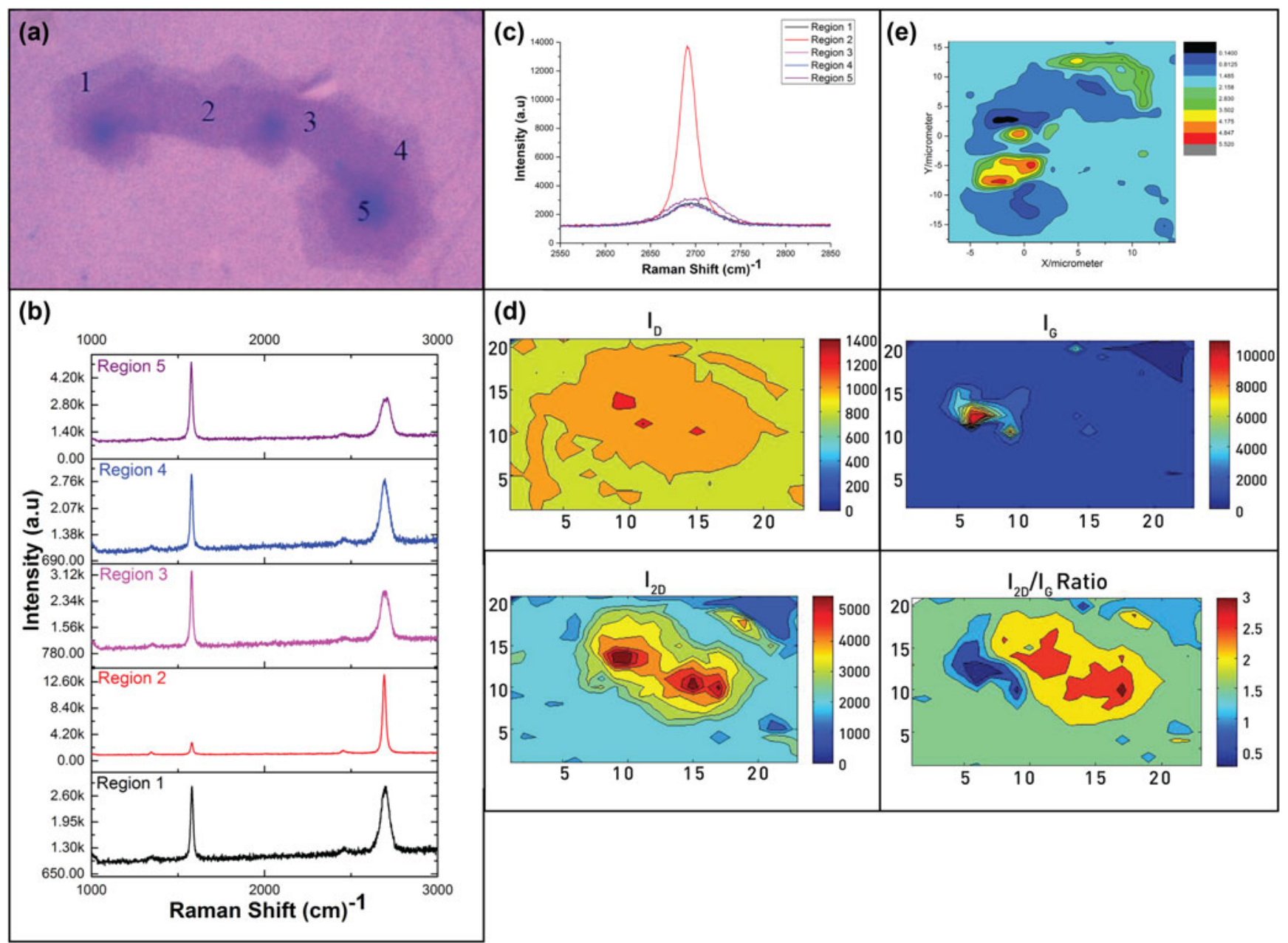

FIG. 5. (a) Marked optical image of graphene grains for Raman spectrum at different points, (b) Raman spectra for Bernal (AB) stack coalesced grains at regions $1,2,3,4$, and 5 , (c) $2 \mathrm{D}$ peak for all the regions, (d) intensity maps for $\mathrm{D}, \mathrm{G}$, and $2 \mathrm{D}$ peaks plotted in matlab and (e) $I_{2 \mathrm{D}} / I_{\mathrm{G}}$ ratio contour mapping in Origin 8.5 software.

graphene layers match with the predicted values of Hao et al. ${ }^{39}$

Figure 4 shows the Raman spectra for two twisted grains combine adjacently. In Fig. 4(a), optical micrograph was marked for different regions of grains. The twisted or misaligned layers significantly affects the mechanical, ${ }^{42}$ thermal, ${ }^{43}$ electrical, ${ }^{44}$ and optical ${ }^{45}$ properties of graphene. The 2D Raman peak shows a complicated behavior for twisted layer graphene. Theoretically, the twisted layers exhibit linear dispersion near the Dirac point with low energy von-Hove singularities. The electronic structure of twisted layers graphene looks similar to single layer but with lesser Fermi velocity, which means, smaller slope of electronic band near $\mathrm{K}$ points. ${ }^{46}$ The orientation angle is very important in twisted layers because it evolves additional symmetry in the form of Moiré superlattice. ${ }^{46,47}$ Moiré superlattice spreads an extra periodicity which imparts a periodic potential and this periodic potential caused changes in the band structure of twisted layers. ${ }^{48,46}$ The change in layer orientation is natural in CVD and causes dispersion in band structure which changes the properties of twisted layers from other stacking. In fact, 2D layered materials have a natural tendency of rotational disorder because van der Waals forces are weak and cannot lock the layers strongly in a particular atomic fashion. So, the individual atomic planes have tendency to slide and rotate with respect to each other.

The 2D Raman peak for regions 1, 2, and 3 appeared around 2687.96, 2689.70, and $2692.83 \mathrm{~cm}^{-1}$ with FWHM values of $25.29,22.34$, and $20.23 \mathrm{~cm}^{-1}$, respectively. There is a consistent decrease in FWHM values of the layers with the increase in number. In comparison with SLG $\left(30.18 \mathrm{~cm}^{-1}\right)$, there is a decrease of $4.89 \mathrm{~cm}^{-1}$ (bilayer), $7.84 \mathrm{~cm}^{-1}$ (trilayer), and $9.95 \mathrm{~cm}^{-1}$ (few layer) in the values of FWHM. The $I_{2 \mathrm{D}} / I_{\mathrm{G}}$ ratio for regions 1,2 , and 3 is $3.42,4.33$, and 5.81, respectively, which is quiet higher than the values of Bernal stacked graphene layers shown in Fig. 3. The higher intensity ratios support the twisted sequence in layers. We observed 
similar finding in the adjacent grain marked as regions 4 , 5,6 , and 7 . The common feature of all the regions is the shape of 2D peak which is symmetrical as compare to Bernal stacked grain. Intensity maps clearly showed different number of layers.

Figure 5 shows a complicated grain structure where three grains coalescing together. The 2D Raman peak for regions 1, 3, 4, and 5 appeared around 2696.76, 2695.20, 2693.83 , and $2699.42 \mathrm{~cm}^{-1}$ with FWHM values of $57.23 \mathrm{~cm}^{-1}$ (trilayer), $61.08 \mathrm{~cm}^{-1}$ (tetralayer), $56.36 \mathrm{~cm}^{-1}$ (trilayer), and $69.39 \mathrm{~cm}^{-1}$ (pentalayer), respectively. ${ }^{39}$ Moreover, the values of $I_{2 \mathrm{D}} / I_{\mathrm{G}}$ ratio for regions $1,3,4$, and 5 is $1.00,0.81,0.96$, and 0.64 , respectively which clearly showed Bernal stacking in the grain. Interestingly, the 2D Raman peak of region 2 shows FWHM value of $20.53 \mathrm{~cm}^{-1}$. The shape of the peak is quite symmetric as compare to other regions and showed $I_{2 \mathrm{D}} / I_{\mathrm{G}}$ ratio of 4.69 hint toward twisted or disoriented stacking of layers. ${ }^{41}$ We believe this region is the area where the grain boundary of the grains are combining which caused change in the orientation of the layers.

\section{CONCLUSION}

The present work is about the synthesis of few layer graphene grains on $\mathrm{Pt}$ foil using CVD. The graphene grains were analyzed in the coalesced condition. Grains of different stacking sequences were observed in the samples, the occurrence of rotational domains are seen to be higher as compare to Bernal domains due to mismatch between Moiré superstructure unit cell and Pt atoms. A systematic characterization was done to understand the stacking sequence in different grains. The preliminary understanding was obtained using optical microscopy. SEM characterization was done to see the magnified form of coalesced grains. Raman spectroscopy clearly showed stacking sequences in the grains when they are merging with each and growing in bigger size. 2D Raman peak of three different coalesced grains were characterized which showed Bernal $\mathrm{AB}$ and twisted layer stacking in the grains. Raman contour mapping also provide imaging of the number of layers which is helpful to get an idea about the number of layers.

\section{ACKNOWLEDGMENTS}

S. Karamat would like to thank TÜBITAK and European Union Marie-Curie Co-Funded 2236 Fellowship for accomplishing this work.

\section{REFERENCES}

1. K.S. Novoselov, A.K. Geim, S.V. Morozov, D. Jiang, Y. Zhang, S.V. Dubonos, I.V. Grigorieva, and A.A. Firsov: Electric field effect in atomically thin carbon films. Science 306, 666-669 (2004).
2. A.K. Geim: Graphene: Status and prospects. Science 324(5934), 1530-1534 (2009).

3. K. Novoselov: Graphene: Materials in the Flatland. Rev. Mod. Phys. 83, 837-849 (2011).

4. F. Bonaccorso, Z. Sun, T. Hasan, and A.C. Ferrari: Graphene photonics and optoelectronics. Nat. Photonics 4, 611-622 (2010).

5. A. Reina, X. Jia, J. Ho, D. Nezich, H. Son, V. Bulovic, M.S. Dresselhaus, and J. Kong: Large area, few-layer graphene films on arbitrary substrates by chemical vapor deposition. Nano Lett. 9, 3087 (2009).

6. X. Li, W. Cai, J. An, S. Kim, J. Nah, D. Yang, R. Piner, A. Velamakanni, I. Jung, E. Tutuc, S.K. Banerjee, L. Colombo, and R.S. Ruoff: Large-area synthesis of high-quality and uniform graphene films on copper foils. Science 324, 1312-1314 (2009).

7. K.S. Kim, Y. Zhao, H. Jang, S. Lee, J.M. Kim, K.S. Kim, J-H. Ahn, P. Kim, J-Y. Choi, and B.H. Hong: Large-scale pattern growth of graphene films for stretchable transparent electrodes. Nature 457, 706-710 (2009).

8. I. Vlassiouk, S. Smirnov, I. Ivanov, P. Fulvio, S. Dai, H. Meyer, M. Chi, D. Hensley, P. Datskos, and N. Lavrik: Electrical and thermal conductivity of low temperature CVD graphene: The effect of disorder. Nanotechnology 22, 275716 (2011).

9. H.S. Song, S.L. Li, H. Miyazaki, S. Sato, K. Hayashi, A. Yamada, N. Yokoyama, and K. Tsukagosh: Origin of the relatively low transport mobility of graphene grown through chemical vapor deposition. Sci. Rep. 2, 1-6 (2012).

10. H. Zhou, W.J. Yu, L. Liu, R. Cheng, Y. Chen, X. Huang, Y. Liu, Y. Wang, Y. Huang, and X. Duan: Chemical vapour deposition growth of large single crystals of monolayer and bilayer graphene. Nat. Commun. 4, 2096 (2013).

11. H. Wang, G. Wang, P. Bao, S. Yang, W. Zhu, X. Xie, and W.J. Zhang: Controllable synthesis of submillimeter single-crystal monolayer graphene domains on copper foils by suppressing nucleation. J. Am. Chem. Soc. 134, 3627 (2012).

12. T. Wu, G. Ding, H. Shen, H. Wang, L. Sun, D. Jiang, X. Xie, and M. Jiang: Triggering the continuous growth of graphene toward millimeter-sized grains. Adv. Funct. Mater. 23, 198 (2013).

13. X. Li, C.W. Magnuson, A. Venugopal, R.M. Tromp, J.B. Hannon, E.M. Vogel, L. Colombo, and R.S. Ruoff: Large-area graphene single crystals grown by low-pressure chemical vapor deposition of methane on copper. J. Am. Chem. Soc. 133, 2816 (2011).

14. L. Gao, W. Ren, H. Xu, L. Jin, Z. Wang, T. Ma, L-P. Ma, Z. Zhang, Q. Fu, L-M. Peng, X. Bao, and H-M. Cheng: Repeated growth and bubbling transfer of graphene with millimetre-size single-crystal grains using platinum. Nat. Commun. 3, 699 (2012).

15. Z. Yan, J. Lin, Z. Peng, Z. Sun, Y. Zhu, L. Li, C. Xiang, E.L. Samuel, C. Kittrell, and L.M. Tour: Toward the synthesis of wafer-scale single-crystal graphene on copper foils. ACS Nano $\mathbf{6}$, 9110 (2012).

16. X. Li, C.W. Magnuson, A. Venugopal, J. An, J.W. Suk, B. Han, M. Borysiak, W. Cai, A. Velamakanni, Y. Zhu, L. Fu, E.M. Vogel, E. Voelkl, L. Colombo, and R.S. Ruoff: Graphene films with large domain size by a two-step chemical vapor deposition process. Nano Lett. 10, 4328-4334 (2010).

17. I. Vlassiouk, M. Regmi, P. Fulvio, S. Dai, P. Datskos, G. Eres, and S. Smirnov: Role of hydrogen in chemical vapor deposition growth of large single-crystal graphene. ACS Nano 5(7), 60696076 (2011).

18. S. Bhaviripudi, X. Jia, M.S. Dresselhaus, and J. Kong: Role of kinetic factors in chemical vapor deposition synthesis of uniform large area graphene using copper catalyst. Nano Lett. 10, 4128 4133 (2010).

19. P. Sutter, J.T. Sadowski, and E. Sutter: Graphene on $\operatorname{Pt}(111)$ : Growth and substrate interaction. Phys. Rev. B 80, 245411 (2009). 
20. P. Merino, M. Svec, A.L. Pinnardi, G. Otero, and J.A. MartinGago: Strain-driven moiré superstructures of epitaxial graphene on transition metal surfaces. ACS Nano 5, 5627-5634 (2011).

21. S. Nie, A.L. Walter, N.C. Bartelt, E. Starodub, A. Bostwick, E. Rotenberg, and K.F. McCarty: Growth from below: Graphene bilayers on $\operatorname{Ir}(111)$. ACS Nano 5(3), 2298-2306 (2011).

22. A.W. Robertson, A. Bachmatiuk, Y.A. Wu, F. Schäffel, B. Rellinghaus, B. Büchner, M.H. Rümmeli, and J.H. Warner: Atomic structure of interconnected few-layer graphene domains. ACS Nano 5, 6610-6618 (2011).

23. R. Rao, N. Pierce, and A.R. Harutyunyanar: Probing the Electrical Properties of Overlapped Graphene Grain Boundaries by Raman spectroscopy, Xiv:1406.1555 [cond-mat.mtrl-sci] (2014).

24. S. Reich and C. Thomsen: Raman spectroscopy of graphite. Philos. Trans. R. Soc., A 362, 2271-2288 (2004).

25. M.S. Dresselhaus and P.C. Eklund: Phonons in carbon nanotubes. Adv. Phys. 49, 705-814 (2000).

26. M.S. Dresselhaus, G. Dresselhaus, R. Saito, and A. Jorio: Raman spectroscopy of carbon nanotubes. Phys. Rep. 409, 47-99 (2005).

27. A.C. Ferrari, J.C. Meyer, V. Scardaci, C. Casiraghi, M. Lazzeri, F. Mauri, S. Piscanec, D. Jiang, K.S. Novoselov, S. Roth, and A.K. Geim: Raman spectrum of graphene and graphene layers. Phys. Rev. Lett. 97, 187401 (2006).

28. L.G. Cancado, A. Reina, J. Kong, and M.S. Dresselhaus: Geometrical approach for the study of $\mathrm{G}^{\prime}$ band in the Raman spectrum of monolayer graphene, bilayer graphene, and bulk graphite. Phys. Rev. B 77, 245408 (2008).

29. W. Fang, A.L. Hsu, R. Caudillo, Y. Song, A.G. Birdwell, E. Zakar, M. Kalbac, M. Dubey, T. Palacios, M.S. Dresselhaus, P.T. Araujo, and J. Kong: Rapid identification of stacking orientation in isotopically labeled chemical-vapor grown bilayer graphene by raman spectroscopy. Nano Lett. 13, 1541 (2013).

30. A.C. Ferrari: Raman spectroscopy of graphene and graphite: Disorder, electron-phonon coupling, doping and nonadiabatic effects. Solid State Commun. 143, 47-57 (2007).

31. A. Das, S. Pisana, B. Chakraborty, S. Piscanec, S.K. Saha, U.V. Waghmare, K.S. Novoselov, H.R. Krishnamurthy, A.K. Geim, A.C. Ferrari, and A.K. Sood: Monitoring dopants by Raman scattering in an electrochemically top-gated graphene transistor. Nat. Nanotechnol. 3, 210 (2008).

32. S. Gayathri, P. Jayabal, M. Kottaisamy, and V. Ramakrishnan: Synthesis of few layer graphene by direct exfoliation of graphite and a Raman spectroscopic study. AIP Adv. 4, 027116 (2014).

33. E.A. Obraztsova, A.V. Osadchy, E.D. Obraztsova, S. Lefrant, and I.V. Yaminsky: Statistical analysis of atomic force microscopy and Raman spectroscopy data for estimation of graphene layer numbers. Phys. Status Solidi B 245(10), 2055 (2008).

34. M. Huang, H. Yan, C. Chen, D. Song, T.F. Heinz, and J. Hone: Phonon softening and crystallographic orientation of strained graphene studied by Raman spectroscopy. Proc. Natl. Acad. Sci. U. S. A. 106, 7304 (2009).

35. K.S. Novoselov, A.K. Geim, S.M. Morozov, M.I. Katsnelson, I.V. Grigorieva, S.V. Dubonos, and A.A. Firsov: Two-dimensional gas of massless Dirac fermions in graphene. Nature 438, 197 (2005).

36. A.K. Geim and K.S. Novoselov: The rise of graphene. Nat. Mater. 6, 183 (2007).

37. E.V. Castro, K.S. Novoselov, S.V. Morozov, N.M.R. Peres, J.M.B. Lopes dos Santos, J. Nilsson, F. Guinea, A.K. Geim, and A.H. Castro Neto: Biased bilayer graphene: Semiconductor with a gap tunable by the electric field effect. Phys. Rev. Lett. 99. 216802 (2007)

38. T. Ohta, A. Bostwick, T. Seyller, K. Horn, and E. Rotenberg: Controlling the electronic structure of bilayer graphene. Science 313, 951 (2006).

39. Y. Hao, Y. Wang, L. Wang, Z. Ni, Z. Wang, R. Wang, C. Keong Koo, Z. Shen, and J.T.L. Thong: Probing layer number and stacking order of few-layer graphene by Raman spectroscopy. Small 6(2), 195-200 (2010).

40. L.M. Malard, J. Nilsson, D.C. Elias, J.C. Brant, F. Plentz, E.S. Alves, A.H. Castro Neto, and M.A. Pimenta: Probing the electronic structure of bilayer graphene by Raman scattering. Phys. Rev. B 76, 201401(R) (2007).

41. L. Liu, H. Zhou, R. Cheng, W.J. Yu, Y. Liu, Y. Chen, J. Shaw, $X$. Zhong, Y. Huang, and X. Duan: High-yield chemical vapor deposition growth of high-quality large-area AB-stacked bilayer graphene. ACS Nano 6(9), 8241-8249 (2012).

42. J. Zhang and J. Zhao: Mechanical properties of bilayer graphene with twist and grain boundaries. J. Appl. Phys. 113, 043514 (2013).

43. A.I. Cocemasov, D.L. Nika, and A.A. Balandin: Phonons in twisted bilayer graphene. Phys. Rev. B 88, 035428 (2013).

44. A. Luican, G. Li, A. Reina, J. Kong, R. Nair, K. Novoselov, A. Geim, and E. Andrei: Single-layer behavior and its breakdown in twisted graphene layers. Phys. Rev. Lett. 106, 126802 (2011).

45. Y. Wang, Z. Ni, L. Liu, Y. Liu, C. Cong, T. Yu, X. Wang, D. Shen, and Z. Shen: Stacking-dependent optical conductivity of bilayer graphene. ACS Nano 4, 4074-4080 (2010).

46. T.E. Beechem, T. Ohta, B. Diaconescu, and J.T. Robinson: Rotational disorder in twisted bilayer graphene. ACS Nano $8(2)$, 1655-1663 (2014).

47. J.M.B. Lopes dos Santos, N.M.R. Peres, and A.H. Castro Neto: Graphene bilayer with a twist: Electronic structure. Phys. Rev. Lett. 99, 256802 (2007).

48. T. Ohta, J.T. Robinson, P.J. Feibelman, A. Bostwick, E. Rotenberg, and T.E. Beechem: Evidence for interlayer coupling and moiré periodic potentials in twisted bilayer graphene. Phys. Rev. Lett. 109, 186807 (2012). 